\title{
Cervical Leiomyosarcoma
}

National Cancer Institute

\section{Source}

National Cancer Institute. Cervical Leiomyosarcoma. NCI Thesaurus. Code C128047.

A malignant neoplasm with smooth muscle differentiation arising from the cervix. 